\title{
Post ICU syndrome among survivors from respiratory critical illness. A prospective study
}

\author{
Ahmad Abbas, Niveen E. Zayed, Samah M. Lutfy
}

Context Post-ICU syndrome (PICS) is a common impairment that develops after critical illness and persists after discharge. It is considered when a new or worsening impairment in physical, cognitive, or mental status develops among survivors from critical illness.

Aim To assess the prevalence of PICS and to define the profile of patients at risk of each domain.

Patients and methods A total of 420 critically ill patients were assessed at the time of ICU discharge for presence of one or more domains of PICS: cognitive dysfunction, psychiatric impairment, and physical disability.

Results A total of 220 (52.4\%) patients without preexisting impairment developed one or more PICS forms. Half of the participants developed cognitive impairment, $14.29 \%$ developed depression, 26.19 developed anxiety, and $35.71 \%$ experienced both muscle weakness and impaired balance. PICS presented in three different patterns: pattern A, with one domain, in which $2.38 \%$ presented with either cognitive or psychiatric affection; pattern B, with two (19.05\%) domains, where 80 patients had cognitive dysfunction, combined with physical affection in 30 patients and psychiatric impairment in 50 patients; and pattern C, with all PICS domains (28.57\%).

\section{Introduction}

Post-ICU syndrome (PICS) is a common impairment that develops after critical illness and persists after discharge from ICU. It is considered when a new or worsening impairment in physical, cognitive, or mental health status develops among survivors from critical illness [1].

Long-term cognitive impairment is reported to occur in $25 \%$ of ICU survivors, a percent that reaches $75 \%$ in a few studies [2]. Risk factors include prior cognitive impairment, prolonged mechanical ventilation (MV), glucose dysregulation, duration of delirium, stroke, alcoholism, hypoxia, hypotension, severe sepsis, and acute respiratory distress syndrome (ARDS) [3].

Moreover, psychological disability (anxiety, depression, and posttraumatic stress disorder) is observed up to $62 \%$ among survivors after discharge from ICU with the same risk factors for cognitive dysfunction in addition to the use of sedation and analgesia in ICU, female sex, lower level of education, and preexisting psychiatric impairment [4-6].

Critical illness neuromuscular weakness is considered the most common physical impairment that occurs in
Multivariate analysis was used to detect independent predictors associated with each domain of PICS.

Conclusion Survivors from critical illness should be screened for different domains of PICS. Cognitive impairment was evident in those with prolonged duration of mechanical ventilation (MV), delirium, stroke, and hypotension.

Psychiatric impairment was evident in females with prolonged sedation and duration of MV, delirium, and hypoglycemia. Physical impairments were evident in those with sepsis, undernutrition, and prolonged duration of MV.

Egypt J Bronchol 2019 13:505-509

(C) 2019 Egyptian Journal of Bronchology

Egyptian Journal of Bronchology 2019 13:505-509

Keywords: anxiety, cognitive dysfunction, depression, muscle weakness, post-intensive care unit syndrome

Department of Chest, Zagazig University, Zagazig, Egypt

Correspondence to Ahmad Abbas, MD, Department of Chest, Zagazig University, Zagazig 44519, Egypt. Tel: +20 115535 5369; e-mail: amabbaas@zu.edu.eg

Received: 25 April 2019 Revised: 15 May 2019 Accepted: 16 July 2019 Published: 25 October 2019

more than quarter of ICU population. Risks include multisystem organ failure, sepsis, prolonged sedation use, and MV ( $>7$ days) $[7,8]$.

This study aimed at assessment of the prevalence of PICS among survivors from respiratory critical illness and to define the profile of patients at risk of each domain.

\section{Patients and methods}

This study was conducted at respiratory ICU (RICU), Zagazig University Hospitals, from January 2017 to January 2019. It included 420 critically ill patients assessed at time of ICU discharge.

\section{Inclusion criteria}

All critically ill patients admitted to RICU for more than $24 \mathrm{~h}$ owing to various diagnoses with no history of neurocognitive impairment during the period of the study and who agreed to participate were included.

This is an open access journal, and articles are distributed under the terms of the Creative Commons Attribution-NonCommercial-ShareAlike 4.0 License, which allows others to remix, tweak, and build upon the work non-commercially, as long as appropriate credit is given and the new creations are licensed under the identical terms. 


\section{Exclusion criteria}

Participants with any of the followings were excluded: malignancy, neuromuscular pathology, pregnancy, and patients discharged on long-term ventilatory support or tracheostomy.

All participants were subjected to the followings:

(1) Good history taking and event documentation during RICU stay with stress on previous ICU admission or $\mathrm{MV}$, respiratory failure, recent ventilatory support and its duration, sepsis, use of sedation, cardiac arrest, ARDS, stroke, delirium, undernutrition, hypoglycemia, hypotension, and hypoxemia.

(2) Assessment of cognitive impairment using Montreal Cognitive Assessment scale, Arabic version. It is a 30-point test in which a score less than 26 points represents a mild cognitive affection [9].

(3) Assessment of psychosocial problems:

(a) The Generalized Anxiety Disorder-7 scale, Arabic version: scores of 5, 10, and 15 are the cut-off points for mild, moderate, and severe anxiety, respectively [10].

(b) Beck Depression Inventory-second edition scale, the Arabic translation: it assesses the presence and severity of depression. Each item is related to how the patient had felt in the previous two weeks. There is a four-point scale for each item ranging from 0 to 3 . Total score of 14-19 represents mild form of depression, 20-28 represents moderate form, and 29-63 represents severe form [11].

(4) Assessment of muscle power strength using Medical Research Council sum score: the sum of the manual muscle test using the Oxford 0-5 scales for the following muscle groups bilaterally shoulder abduction, elbow flexion, wrist extension, hip flexion, knee extension, and dorsiflexion. A score of less than 48/ is consistent with ICUacquired weakness [12].

(5) Balance evaluation using Berg Balance Scale. Score of less than $45 / 58$ indicates an increased risk for falls [13].

\section{Ethical statement}

The study was approved by Ethics Committee of Faculty of Medicine, Zagazig University. A written informed consent was obtained from all participants.

\section{Statistical analysis}

All statistics were performed using Minitab 17.1.0.0 for Windows (2013; Minitab Inc., State College, PA,
USA). Continuous variables were presented as mean (SD) and nonparametric data as number (\%). Multivariate logistic regression analysis with stepwise elimination was used to determinate the independent predictors of PICS domains. $P$ value above 0.05 was considered significant.

\section{Results}

This study enrolled 695 patients; of them, 420 patients completed the study, as 75 patients were excluded according to the exclusion criteria, 80 patients died before discharge, and 95 patients refused to participate. A total of 420 survivors from critical illness were evaluated at the time of ICU discharge. Table 1 demonstrated the prevalence of different domains of PICS. Overall, 220 (52.4\%) patients without preexisting impairment developed one or more PICS forms. Half of the participants developed cognitive impairment, 14.29\% developed depression, 26.19\% developed anxiety, and finally, 35.71\% experienced both muscle weakness and impaired balance.

Different patterns of PICS were shown in Table 2. PICS presented in three different patterns: pattern A, with one domain, in which $2.3 \%$ presented with either cognitive or psychiatric affection; pattern $\mathrm{B}$, with two

Table 1 Prevalence of different domains of post-intensive care unit syndrome

\begin{tabular}{lcc}
\hline Post-ICU syndrome domain & \multicolumn{2}{c}{ Total $(N=420)$} \\
\hline Cognitive dysfunction & 210 & 50 \\
Depression & 60 & 14.29 \\
Anxiety & 110 & 26.19 \\
Muscle weakness & 150 & 35.71 \\
Balance affection & 150 & 35.71 \\
\hline
\end{tabular}

Categorical data represented as $n(\%)$.

Table 2 Patterns of post-intensive care unit syndrome

\begin{tabular}{lcc}
\hline Post-ICU syndrome patterns & \multicolumn{2}{c}{ Total $(N=420)$} \\
\hline Free & 200 & 47.62 \\
A pattern & 20 & 4.76 \\
Cognitive dysfunction & 10 & 2.38 \\
Physical disorder & 10 & 2.38 \\
B pattern & 80 & 19.05 \\
Cognitive dysfunction & 80 & 19.05 \\
Physical disorder & 30 & 7.14 \\
Psychic disorder & 50 & 11.90 \\
C pattern & 120 & 28.57 \\
Cognitive dysfunction & 120 & 28.57 \\
Physical disorder & 120 & 28.57 \\
Psychic disorder & 120 & 28.57 \\
\hline
\end{tabular}

Categorical data represented as $n(\%)$. A pattern, patients with one form of post-ICU disorders; B pattern, patients with two forms of post-ICU disorders; C pattern, patients with all forms of postICU disorders. 
(19.05\%) domains, where 80 patients had cognitive dysfunction, combined with physical affection in 30 patients and psychiatric impairment in 50 patients; and pattern C, with all PICS domains (28.57\%).

Demographic and risk factors of patients with PICS are shown in Table 3. In this study, patients at risk of PICS had mean age of $51.23 \pm 10.07$ years, $65 \%$ were males, previously ICU admitted were $31.82 \%$, and previously mechanically ventilated were $9.09 \%$. Recent institution of ventilatory support owing to respiratory failure was observed in $95.45 \%$, with mean duration of MV of 9.82 \pm 4.21 days. Sepsis was found in $13.64 \%$, prolonged sedation in $36.36 \%$, cardiac arrest in $4.55 \%$, ARDS in $13.64 \%$, stroke in $18.18 \%$, delirium in $45.45 \%$, poor nutrition in $45.45 \%$, hypoglycemia in $18.18 \%$, and finally hypotension in $22.73 \%$.

Multiple regression analysis of variables associated with each domain of PICS is shown in Tables 4-6. Multiple regression analysis of variables associated with post-ICU cognitive dysfunction revealed four independent predictors: duration of $\mathrm{MV}$ [odds ratio $(\mathrm{OR})=1.19$, confidence interval $(\mathrm{CI})=1.1-1.28, P<0.001)$, stroke $(\mathrm{OR}=2.52, \quad \mathrm{CI}=0.95-6.69, \quad P=0.038), \quad$ delirium $(\mathrm{OR}=3.21, \quad \mathrm{CI}=1.76-5.84, \quad P<0.001), \quad$ and hypotension $(\mathrm{OR}=3.44, \mathrm{CI}=1.52-7.67, \quad P=0.002)$. Multiple regression analysis of variables associated with post-ICU psychiatric disorder revealed five independent predictors: duration of $\mathrm{MV}(\mathrm{OR}=0.80$, $\mathrm{CI}=0.669-0.946, \quad P=0.005), \quad$ prolonged use of sedation $(\mathrm{OR}=11.50, \quad \mathrm{CI}=24.55-52.64, \quad P<0.001)$,

Table 3 Demography and risk factors of patients with postintensive care unit syndrome

\begin{tabular}{lcc}
\hline Characteristics & \multicolumn{2}{c}{ Total $(N=220)$} \\
\hline Age & 51.23 & 10.07 \\
Sex (male) & 143 & 65 \\
Previous ICU admission (yes) & 70 & 31.82 \\
Previous MV & 20 & 9.09 \\
Recent MV & 210 & 95.45 \\
Duration of MV use & 9.82 & 4.21 \\
Sepsis & 30 & 13.64 \\
Prolonged sedation & 80 & 36.36 \\
Respiratory failure & 210 & 95.45 \\
Cardiac arrest & 10 & 4.55 \\
ARDS & 30 & 13.64 \\
Stroke & 40 & 18.18 \\
Delirium & 100 & 45.45 \\
Undernutrition & 100 & 45.45 \\
Hypoglycemia & 40 & 18.18 \\
Hypotension & 50 & 22.73 \\
Hypoxemia & 210 & 95.45 \\
\hline ARDS. & &
\end{tabular}

ARDS, acute respiratory distress syndrome; MV, mechanical ventilation. Continues data were represented as mean and SD, categorical data as $n(\%)$. delirium $\quad(\mathrm{OR}=4.92, \quad \mathrm{CI}=2.094-11.55, \quad P<0.001)$, hypoglycemia $(\mathrm{OR}=9.96, \mathrm{CI}=3.068-32.31, P=0.02)$, and female sex $(\mathrm{OR}=0.01, \quad \mathrm{CI}=0.005-0.0349$, $P=0.01$ ). Multiple regression analysis of variables associated with post-ICU physical disorder revealed three independent risk factors: duration of $\mathrm{MV}$ $(\mathrm{OR}=1.35, \quad \mathrm{CI}=1.2073-1.517, \quad P<0.001)$, undernutrition $\quad(\mathrm{OR}=0.88, \quad \mathrm{CI}=0.393-1.963$, $P=0.015)$, and sepsis $(\mathrm{OR}=6.28, \mathrm{CI}=1.323-29.801$, $P=0.02)$

\section{Discussion}

In the current study, among 420 survivors enrolled and assessed at the time of discharge from ICU, 200 (47.6\%) patients were free from PICS, whereas 220

Table 4 Multiple regression analysis of variables associated with post-intensive care unit cognitive dysfunction

\begin{tabular}{lccc}
\hline Factors & OR & $95 \% \mathrm{Cl}$ & $P$ \\
\hline Age & 0.99 & $(0.9656,1.0167)$ & 0.48 \\
Duration of MV use & 1.19 & $(1.1019,1.2887)$ & $<0.001$ \\
Stroke & 2.52 & $(0.9504,6.6978)$ & 0.038 \\
Delirium & 3.21 & $(1.7657,5.8448)$ & $<0.001$ \\
Hypoglycemia & 3.55 & $(1.4006,8.9820)$ & 0.07 \\
Hypotension (septic shock) & 3.44 & $(1.5205,7.7645)$ & 0.002 \\
\hline
\end{tabular}

Pearson; $\chi^{2}=404.6, P=0.5$, the model was adjusted for all variables using stepwise elimination. $\mathrm{Cl}$, confidence interval; MV, mechanical ventilation; OR, odds ratio. $P$ value considered significant if less than 0.05 .

Table 5 Multiple regression analysis of variables associated with post-intensive care unit psychiatric disorder

\begin{tabular}{lccc}
\hline Factors & OR & $95 \% \mathrm{Cl}$ & $P$ \\
\hline Duration of MV use & 0.80 & $(0.6696,0.9465)$ & 0.005 \\
Prolonged sedation & 11.50 & $(24.5536,52.6416)$ & $<0.001$ \\
Delirium & 4.92 & $(2.0949,11.5522)$ & $<0.001$ \\
Hypoglycemia & 9.96 & $(3.0686,32.3122)$ & 0.02 \\
Sex (female) & 0.01 & $(0.0051,0.0349)$ & 0.01 \\
\hline
\end{tabular}

Pearson; $\chi^{2}=203.4, P=1$, the model was adjusted for all variables using stepwise elimination. $\mathrm{Cl}$, confidence interval; $\mathrm{MV}$, mechanical ventilation; $\mathrm{OR}$, odds ratio. $P$ value considered significant if less than 0.05 .

Table 6 Multiple regression analysis of variables associated with post-intensive care unit physical disorder

\begin{tabular}{lccc}
\hline Factors & OR & $95 \% \mathrm{Cl}$ & $P$ \\
\hline Age & 0.97 & $(0.9367,0.9946)$ & 0.2 \\
Previous ICU admission & 2.22 & $(0.9760,5.0288)$ & 0.06 \\
Previous MV support & 3.44 & $(0.4460,26.6083)$ & 0.23 \\
Duration of MV use & 1.35 & $(1.2073,1.5175)$ & $<0.001$ \\
Undernutrition & 0.88 & $(0.3932,1.9639)$ & 0.015 \\
Sepsis & 6.28 & $(1.3238,29.8016)$ & 0.02 \\
\hline
\end{tabular}

Deviance; $\chi 2=258.7, P=1$, the model was adjusted for all variables using stepwise elimination. $\mathrm{Cl}$, confidence interval; MV, mechanical ventilation; $\mathrm{OR}$, odds ratio. $P$ value considered significant if less than 0.05 . 
(52.4\%) patients without preexisting impairment developed one or more PICS forms.

Half of the participants developed post-ICU cognitive impairment, $14.29 \%$ developed depression, $26.19 \%$ developed anxiety, and finally $35.71 \%$ experienced both ICU muscle weakness and impaired balance.

In a study by Marra et al. [14], one or more PICS problems were present in $64 \%$ and $56 \%$ after 3 months and 1 year after discharge, respectively. Of patients assessed, 38\% had cognitive dysfunction, 26\% had physical weakness, and $33 \%$ had depressive impairment. At 12 months, 33\% had cognitive impairment, $21 \%$ had disability, and 31\% had depression.

One study reviewed the medical records of patients referred for psychiatric consultation and screening of cognitive dysfunction or mood affection at discharge from the ICU during the 12 -month period, and $43.3 \%$ screened positive for cognitive impairment and 60\% experienced depressive symptoms [15].

Jones et al. [16] reported that $86.7 \%$ of patients one week after discharge from ICU showed evidence of strategic thinking and problem-solving impairment.

In Torgersen et al. [17], 64.3\% of enrolled patients had a cognitive impairment at time of ICU discharge.

Global cognition and executive function were assessed in the study by Pandharipande et al. [18] 3 and 12 months after discharge on 821 adults with respiratory failure or shock. Overall, $6 \%$ of their participants had cognitive impairment at baseline. After 3 months, 40\% of participants had cognitive score similar to patients with moderate traumatic brain injury (1.5 SD), and $26 \%$ had scores similar to patients with mild Alzheimer's disease (2 SD).

In the current study, PICS presented in three different patterns: pattern $\mathrm{A}$, which had one domain, in which 2.3\% presented with either cognitive or psychiatric affection; pattern B, with two domains (19.05\%), where 80 patients had all cognitive dysfunction combined with physical affection in 30 patients and psychiatric impairment in 50 patients; and pattern C, which developed all PICS domains (28.57\%).

Marra et al. [14] reported that co-occurrence of two or more PICS domains was present in $25 \%$ of patients at 3 months and $21 \%$ of them after 1 year of discharge. All domains of PICS were present in only 6 and $4 \%$ at 3 and 12 months, respectively.
In this study, patients at risk of PICS had mean age of $51.23 \pm 10.07$ years, $65 \%$ were males, previously ICU admitted patients represented $31.82 \%$, and previously mechanically ventilated patients represented 9.09\%. Recent institution of ventilatory support owing to respiratory failure was observed in $95.45 \%$, with mean duration of MV of $9.82 \pm 4.21$ days. Sepsis was found in $13.64 \%$, prolonged sedation in $36.36 \%$, cardiac arrest in $4.55 \%$, ARDS in $13.64 \%$, stroke in $18.18 \%$, delirium in $45.45 \%$, poor nutrition in $45.45 \%$, hypoglycemia in $18.18 \%$, and finally hypotension in $22.73 \%$.

Marra et al. [14] found that survivors who were free from PICS tended to be younger, more educated, less frail, and had fewer comorbidities than those with PICS. Severity of illness on admission was similar between those with or without PICS. Patients who were mechanically ventilated, developed sepsis, coma, or delirium during ICU course were more likely to have PICS.

In comparison with studies that assessed PICS in general ICUS, our results agreed that more than five of every 10 patients developed PICS and the majority developed cognitive impairment. On the contrary, in general ICU, the majority developed one domain of PICS and was suggested to be a sequela of severe critical illness rather than part of syndrome, whereas in the current work, more than one in every four patients developed PICS with all three domains. This difference can be attributable to the following: first, different patient characteristics in RICU, in which $95 \%$ of the studied population had respiratory failure and blood gases disturbances in addition to share a high percent of other accusing factors of PICS with general ICUS, and second, the different enrolling criteria and PICS definition between studies. In this study, only new insults were taken into consideration, and patients were included on the basis of not having a previous defect compared with other studies that included those with new or worsened previous dysfunction $[1,5,7]$.

Multiple regression analysis of variables associated with post-ICU cognitive dysfunction revealed four independent predictors: duration of $\mathrm{MV}$, stroke, delirium, hypotension (Table 4). Chung et al. [15] in their study pointed contrary results, in which post-ICU cognitive impairment was not associated with the usual risk factors including age, preexisting cognitive impairment, and duration of delirium in the ICU but was associated with the initial severity of illness assessed by SOFA score. 
Pandharipande et al. [18] concluded that persistent worsened cognition was independently associated with longer duration of delirium, a finding that was not influenced by sedation or analgesia, age, preexisting cognition, and organ failures during ICU course.

Multiple regression analysis of variables associated with post-ICU psychiatric disorder revealed five independent predictors: duration of $\mathrm{MV}$, prolonged use of sedation, delirium, hypoglycemia, and female sex (Table 5).

Chung et al. [15] in their report found a significant association between female sex (88.9\%) and postcritical illness depressive disability and trends of association between post-ICU memories and duration of ventilatory support and ICU length of stay.

Davydow et al. [19] in their systematic review included 1213 patients and reported a prevalence of post-critical illness significant depression in 28\%. Neither sex nor age was an independent risk factor. Moreover, severity of illness on admission was not a risk factor. However, early post-ICU depressive symptoms were a strong predictor for subsequent depression.

Multiple regression analysis of variables associated with post-ICU physical disorder revealed three independent risk factors: duration of $\mathrm{MV}$, undernutrition, and sepsis (Table 6).

Regarding study limitation, this study assessed shortterm acute effects of surviving a critical care illness as all evaluation was done at the time of discharge, but longterm effects that might persist cannot be assessed owing to difficult follow-up and recruitment of survivors in multiple assessment visits.

\section{Conclusion}

Survivors from critical illness should be screened for different domains of PICS. Half of participants in the current work experienced post-ICU cognitive impairment, which was evident in those with prolonged duration of $\mathrm{MV}$, delirium, stroke, and hypotension. Moreover, 14.29 and 26.19\% were reported to have depression and anxiety, respectively, which were more evident in females with prolonged duration of MV and sedation, delirium, and hypoglycemia. Finally, post-ICU physical impairments were reported in $35.71 \%$, which was evident in those with sepsis, undernutrition, and prolonged duration of MV.

\section{Financial support and sponsorship}

Nil.

\section{Conflicts of interest}

There are no conflicts of interest.

\section{References}

1 Needham DM, Davidson J, Cohen H, Hopkins RO, Weinert C, Wunsch H et al. Improving long-term outcomes after discharge from intensive care unit: report from a stakeholders' conference. Crit Care Med 2012; 40:502-509.

2 Needham DM, Dinglas VD, Morris PE, Jackson JC, Hough CL, MendezTellez PA, et al. Physical and cognitive performance of patients with acutelung injury 1 year after initial trophic versus full enteral feeding.EDEN trial follow-up. Am J Respir Crit Care Med 2013; 188:567-576.

3 Iwashyna TJ, Ely EW, Smith DM, Langa KM. Long-term cognitive impairment and functional disability among survivors of severe sepsis. JAMA 2010; 304:1787-1794.

4 Hopkins RO, Weaver LK, Collingridge D, Parkinson RB, Chan KJ, Orme JF Jr. Two-year cognitive, emotional, and quality-of-life outcomes in acute respiratory distress syndrome. Am J Respir Crit Care Med 2005; 171:340-347.

5 Wunsch H, Christiansen CF, Johansen MB, Olsen M, Ali N, Angus DC, et al. Psychiatric diagnoses and psychoactive medication use among nonsurgical critically ill patients receiving mechanical ventilation. JAMA 2014; 311:1133-1142.

6 Jackson JC, Pandharipande PP, Girard TD, Brummel NE, Thompson JL, Hughes CG, et al. Depression, post-traumatic stress disorder, and functional disability in the BRAIN-ICU study: a longitudinal cohort study. Lancet Respir 2014; 2:369-379.

7 Needham DM, Wozniak AW, Hough CL, Morris PE, Dinglas VD, Jackson $\mathrm{JC}$, et al. Risk factors for physical impairment after acute lung injury in a national, multicenter study. Am J Respir Crit Care Med 2014 189:1214-1224.

8 Fletcher SN, Kennedy DD, Ghosh IR, Misra VP, Kiff K, Coakley JH, et al. Persistent neuromuscular and neurophysiologic abnormalities in long term survivors of prolonged critical illness. Crit Care Med 2003; 31:1012-1016.

9 Smith T, Gildeh N, Holmes C. The Montreal Cognitive Assessment: validity and utility in a memory clinic setting. Can J Psychiatry 2007; 52:329-332.

10 Spitzer RL, Kroenke K, Williams JB, Löwe B. A brief measure for assessing generalized anxiety disorder: the GAD-7. Arch Intern Med 2006 166:1092-1097.

11 Beck AT, Steer RA, Brown GK. Beck Depression Inventory manual. 2nd ed. San Antonio, TX: Psychological Corporation; 1996.

12 Ciesla N, Dinglas V, Fan E, Kho M, Kuramoto J, Needham D. Manual muscle testing: a method of measuring extremity muscle strength applied to critically ill patients. J Vis Exp 2011; 12:pii:2632.

13 Denehy L, Nordon-Craft A, Edbrooke L. Outcome measures report different aspects of patient function three months following critical care. Intensive Care Med 2014; 40:1862-1869.

14 Marra A, Pandharipande PP, Girard TD, Patel MB, Hughes CG, Jackson $\mathrm{JC}$, et al. Cooccurrence of post-intensive care syndrome problems among 406 survivors of critical illness. Crit Care Med 2018; 46:1393-1401.

15 Chung CR, Yoo HJ, Park J, Ryu S. Cognitive impairment and psychological distressat discharge from intensive care unit. Psychiatry Investig 2017; 14:376-379.

16 Jones C, Griffiths RD, Slater T, Benjamin KS, Wilson S. Significant cognitive dysfunction in non-delirious patients identified during and persisting following critical illness. Intensive Care Med 2006; 32:923-926.

17 Torgersen J, Hole JF, Kvale R, Wentzel-Larsen T, Flaatten H. Cognitive impairments after critical illness. Acta Anaesthesiol Scand 2011; 55:1044-1051

18 Pandharipande PP, Girard TD, Jackson JC, Morandi A, Thompson JL, Pun $\mathrm{BT}$, et al. Long-term cognitive impairment after critical illness. $N$ Engl $J$ Med 2013; 369:1306-1316.

19 Davydow DS, Gifford JM, Desai SV, Bienvenu OJ, Needham DM. Depression in general intensive care unit survivors: a systematic review. Intensive Care Med 2009; 35:796-809. 